\title{
Flow Actuation by DC Surface Discharge Plasma Actuator in Different Discharge Modes
}

\author{
Yeon-Sung Kim* and Jichul Shin** \\ Department of Aerospace Engineering, University of Ulsan, Ulsan 44611, Korea
}

\begin{abstract}
Aerodynamic flow control phenomena were investigated with a low-current DC surface discharge plasma actuator. The plasma actuator was found to operate in three different discharge modes with similar discharge currents of about $1 \mathrm{~mA}$ or less. Stable continuous DC discharge without audible noise was obtained at higher ballast resistances and lower discharge currents. However, even with continuous DC power input, a low-frequency self-pulsed discharge was obtained at lower ballast resistances, and a high-frequency self-pulsed discharge was obtained at higher set-point currents and higher ballast resistances, both with audible noise. The Schlieren image reveals that the low-frequency self-pulsed mode produces a synthetic jet-like flow implying that a gas heating effect plays a role, even though the discharge current is small. The highfrequency self-pulsed mode produces pulsed jets in a tangent direction, and the continuous DC mode produces a steady straight pressure wave. Particle image velocimetry (PIV) images reveal that the induced flow field by the low-frequency selfpulsed mode has flow propagating in the radial direction and centered between the electrodes. The high-frequency selfpulsed mode and continuous DC mode produce flow from the anode to the cathode. The perturbed region downstream of the cathode is larger in the high-frequency self-pulsed mode with similar maximum speeds.
\end{abstract}

Key words: flow control, plasma actuator, self-Pulsed, DC discharge

\section{Introduction}

Aerodynamic flow control is an interesting topic in aerospace engineering and has been studied for a long time. Increasing attention is being focused on unmanned aerial vehicles (UAVs) [1], and appropriate flow control technology is needed. Typical UAVs can be simply classified into two groups: those with small bodies operated at low altitudes [2] and those with bigger bodies operated at high altitudes, which are called HALE (high altitude long endurance) UAVs [3]. They generally fly in low Reynolds-number (Re\#) regimes ranging from tens of thousands to hundreds of thousands corresponding to laminar flow.

UAVs are becoming more versatile and being equipped with multiple functions as technology advances. UAVs need a wide range of aerodynamic performance for a variety of required missions, but because laminar flow is very sensitive to unsteady disturbances compared with turbulent flow, it can be greatly affected by small fluctuations on the surfaces [4]. In particular, due to the lack of a bulk mixing mechanism, laminar flow tends to cause flow separation more easily than turbulent flow. Hence, studies on laminar flow mostly focus on laminar separation phenomena. The choice of airfoil can greatly affect laminar flow separation [5], and the shape of the wing can also play a role [6]. However, these considerations do not accommodate dynamic changes occurring in flight situations. Hence, either passive or active flow control capability that is applicable to various flight situations is more effective.

Many studies on passive flow control have been performed [7-12]. Examples include surface roughness control and vortex generators. Passive flow control has a bigger on the flow than active control. However, its disadvantages include increased weight, drag, noise, and vibration [7]. In contrast,
This is an Open Access article distributed under the terms of the Creative Commons Attribution Non-Commercial License (http://creativecommons.org/licenses/by$\mathrm{nc} / 3.0 /$ which permits unrestricted non-commercial use, distribution, and reproduction in any medium, provided the original work is properly cited. (c) 
active control works in a transient manner when needed [13, 14]. Blowing jets [15] and morphing wings [16] are examples of active flow control.

Plasma flow-control techniques continue to gain attention as a replacement for conventional methods of mechanical flow control. Flow control using a plasma actuator can be utilized on the wide surface of a wing without moving mechanical parts in a wide range of flow conditions because of high-bandwidth actuation [17]. Plasma flow control can be especially practical in low-Re\# flows at high altitudes. The effectiveness of a mechanical control surface decreases with air density, and a plasma actuator is less affected by the ambient air than at low altitudes where it can be hindered by weather conditions.

Various types of plasma flow actuators are possible, depending on the types of discharges used. Examples include dielectric-barrier discharge, glow discharge, and arc discharge [18]. Dielectric-barrier discharge (DBD) plasma actuators are driven by AC input at several $\mathrm{kHz}$ and are the most commonly used in low-speed flows [19-26]. However, the actuation effect of DBD actuators generally produces asymmetric forcing due to the oscillatory power input [27]. In addition, the actuation capacity is limited by an increased gas heating effect at higher discharge currents. Several efforts have been made to overcome these disadvantages by adding a DC shift to the AC power input [28] or by using a meshed electrode [29].

In contrast to DBD plasma flow actuators, DC plasma actuators can provide unidirectional flow actuation owing to the fixed polarity of the electrodes. Depending on the purpose of flow actuation, an electrostatic forcing effect or gas heating effect can be utilized. In low-Re\# flows, corona discharge [30] or DC glow discharge [31] can be used. These methods fundamentally rely on an electrostatic forcing effect rather than a gas heating effect, and the actuation effect scales with the discharge current up to a certain current level, above which the gas heating effect becomes significant.

It is natural to expect that with guaranteed temporal uniformity of the DC discharge, the actuation effect achieved will be steady with a continuous DC power input unless a gas heating effect continuously builds up. However, in recent studies, we found that the flow actuation effect can differ in specific discharge conditions, even though the provided DC power is unchanged. For example, a discharge in certain conditions produced an isotropic expansion, even with low discharge currents. This is likely to be achieved by the gas heating effect of low-current DC discharge plasma, which is not expected to occur in general.

This paper reports three different discharge modes and corresponding flow actuation effects achieved by a low- current DC discharge plasma actuator. High-speed Schlieren and particle image velocimetry (PIV) techniques provide detailed information about the structures of the induced flow velocity field in different actuation modes. Currentvoltage (I-V) characteristics of the discharges in each mode are provided to help understand the flow actuation mechanisms.

\section{Experiment Setup}

\subsection{DC plasma actuator and electrical connection}

Figure 1 shows the circuit diagram and schematic configuration of the experimental setup of the DC plasma actuator. Sharp-edged nickel electrodes (one anode and one cathode) were placed facing each other on a flat mica dielectric. The electrodes were tightly glued to the dielectric using epoxy, and methanol was used to clean the impurities. To avoid unnecessary disturbance to an induced flow caused by an irregular surface, the actuator and electrode assembly were sufficiently flattened. Three different gaps of $1 \mathrm{~mm}, 2.5$ $\mathrm{mm}$, and $5 \mathrm{~mm}$ between the anode and cathode were used.

The plasma actuator was placed inside a vacuum chamber made with acrylic plates to provide a quiescent environment for characterizing the actuation performance without disturbance from ambient air flows. To test the effectiveness of the plasma actuator in low-Re\# flows, it was mounted

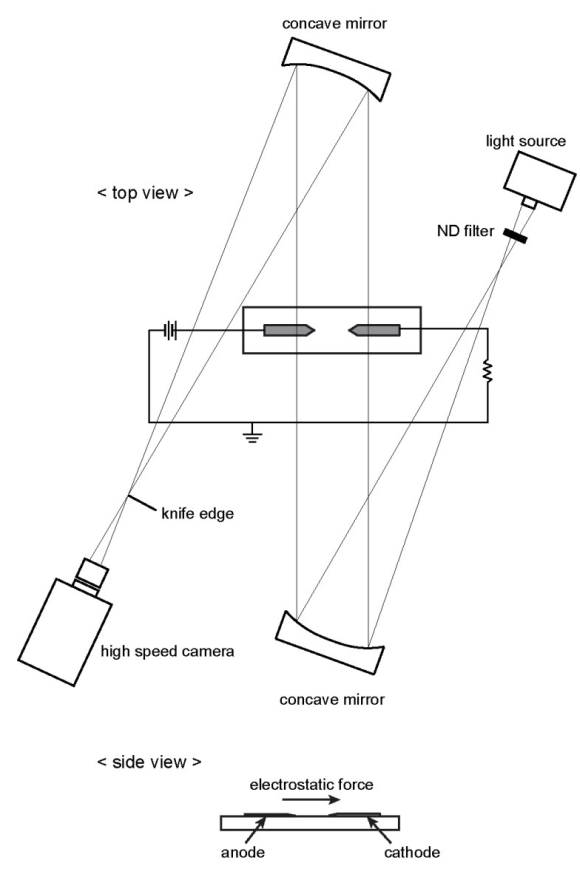

Fig. 1. Diagram of plasma actuator and experiment setup with schlieren system. 
at $1 / 4$ chord on top of an NACA0012 airfoil and tested in a visualization wind tunnel. The discharge was powered by a continuous DC power supply that can provide voltage and current up to $15 \mathrm{kV}$ and $20 \mathrm{~mA}$, respectively. The discharge regime was controlled by varying the ballast resistances and discharge currents. Six different resistance values of $1 \mathrm{k} \Omega, 220$ $\mathrm{k} \Omega, 5 \mathrm{M} \Omega, 10 \mathrm{M} \Omega, 15 \mathrm{M} \Omega$, and $20 \mathrm{M} \Omega$ were used as ballast to adjust the discharge operating conditions, which affect the discharge regime and hence modify the flow actuation characteristics. The current waveform was monitored and recorded by measuring the voltage drop across a currentview resistor ( $10 \Omega$ or $1 \mathrm{k} \Omega$ ) using a $500-\mathrm{MHz}$ oscilloscope (LeCroy LT).

\subsection{Flow visualization}

The flow field induced or modified by the plasma actuator was visualized by Schlieren imaging and PIV measurement. A high-power white light source provided continuous lighting for the Schlieren imaging, and a high-speed camera (Photron SA120K) was used to capture the flow field images at a frame rate of up to $5 \mathrm{kHz}$. A vertical knife edge was used to increase the Schlieren sensitivity in the stream-wise direction parallel to the actuator surface.

The detailed flow field near the plasma actuator was obtained by the PIV technique. A 200-mJ pulsed Nd:Yag laser (Evergreen 200) triggered by a pulse delay generator (Quantum composer 9528) illuminated $\mathrm{TiO}_{2}$ seed particles, and scattered images were collected by a double-exposure CDD camera (PCO 1400). The laser beams were attenuated by a neutral density filter and passed through one cylindrical lens and one spherical lens to provide sheet beams with a thickness of less than $1 \mathrm{~mm}$. To avoid particle agglomeration, the $\mathrm{TiO}_{2}$ particles were baked in an oven before being seeded. Particles were seeded inside the evacuated vacuum chamber, and the electrical power to the actuator was turned on when the seed particles were nearly stationary at uniform density. Fused silica windows were installed on both sides of the vacuum chamber for laser penetration.

\section{Result and discussion}

\subsection{Operating modes of DC surface discharge plas- ma actuator}

Figure 2 shows photographs of the smoke visualization of the flow field around the NACA0012 symmetric airfoil when the plasma actuator was turned off (a) and turned on (b) at certain flow and discharge conditions. One pair of plasma actuators was placed at $1 / 4$ of the chord length on the upper surface. The electrode gap distance was $1 \mathrm{~mm}$, and the discharge current was $0.5 \mathrm{~mA}$. The left image (a) shows a wellattached flow field when the plasma actuator was not turned on. However, when the plasma actuator was turned on (b), the streamline near the actuator deviated significantly, and as a result, the far-field streamlines were pushed upward. This has not usually been observed in many similar studies $[32,33]$. Note that the electrode is aligned with the free stream direction and the anode is placed upstream so that the electric field lines can be aligned in the downstream direction. In this situation, the general expectation regarding flow actuation by the DC discharge (unless a gas heating effect is significant) is that the induced momentum will increase from the anode to the cathode, which is expected to promote further acceleration. However the case shown in Fig. 2 (b) exhibits an adverse effect on the boundary layer flow by causing turbulent mixing in the vertical direction.

To understand this actuation phenomenon in detail, the discharge characteristics of the DC surface glow discharge were investigated. Fig. 3 shows photographs of the discharge structures produced by the DC surface plasma actuator at various operating conditions. The gap distance between the electrodes is $1 \mathrm{~mm}$, and the left electrode is the anode. The images were filtered to show the discharge structure more clearly. The left image corresponds to the low-ballast case $(220 \mathrm{k} \Omega)$, while the middle and the right images correspond to high-ballast cases $(10 \mathrm{M} \Omega)$. The discharge current for case (b) is set at about $0.2 \mathrm{~mA}$, which is lower than that for case (c), where the discharge current is about $0.7 \mathrm{~mA}$.

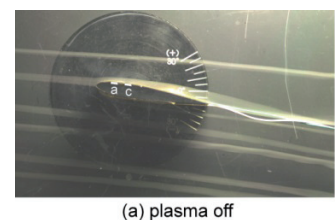

(a) plasma off

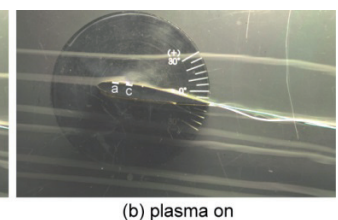

(b) plasma on
Fig. 2. Smoke flow visualization of flow field around NACA 0012 airfoil. (a) shows smoke traces when the plasma actuator is turned off. (b) shows perturbed flow field produced by DC surface glow discharge plasma actuator. One pair of electrodes is placed at the indicated location. $1 \mathrm{~mm}$ electrode gap distance with $10 \mathrm{M} \Omega$ ballast resistance. The discharge current is set at $0.5 \mathrm{~mA}$.

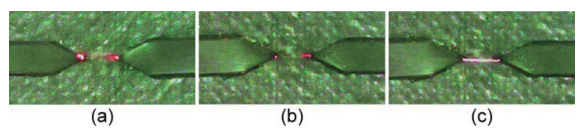

Fig. 3. Photo images of DC discharge structures. Left electrode is cathode and right one is anode in each case. Gap distance between electrodes is $1 \mathrm{~mm}$. (a) corresponds to a low ballast (200 k $\Omega$ ) case and (b) and (c) correspond to high ballast (10 M $\Omega$ ) cases. The discharge current in the case (b) is lower than that in the case (c). 
At high pressures near atmospheric pressure, it is hard to sustain a stable DC glow discharge with a conventional plate-to-plate geometry. Instabilities near the exposed electrodes can easily cause glow-to-arc transition under these conditions [34]. In addition, the operating regime of the DC discharge is determined by external loads [35]. As shown in Fig. 3, the discharge structures are slightly different, and three typical structures occur repeatedly over many runs in similar conditions. Case (a) exhibits brighter glow near both the cathode and anode regions, and the bulk region between the electrodes is vaguely luminous. Case (b) exhibits brighter glow mostly near the anode region. Case (c) exhibits constricted glow discharge stretched between the electrodes.

Case (a) and case (b) may look similar, but interestingly, the discharge in case (a) produces an audible sound while case (b) is quiet as in a Townsend discharge, even at similar discharge currents. Case (c) produces a high-frequency noise at higher currents than case (b). Similar behaviors are also observed with larger electrode gaps. It turns out that the structural differences of the discharge are caused by the distinctive electrical characteristics that depend on ballast resistances and discharge currents.

Figure 4 presents the current waveforms measured downstream of the discharges corresponding to the cases in Fig. 3 (mode (a), mode (b), and mode (c)). The waveform of mode (a) occurs when the ballast resistance is low (220 $\mathrm{k} \Omega$ ). The discharge current overshoots when the discharge is turned on and shuts itself off in order to maintain the average set-point current of $1 \mathrm{~mA}$. Because the ballast resistance is low, the discharge plasma is capable of drawing higher currents for a given supply voltage. But the current is controlled by the power source to maintain the discharge
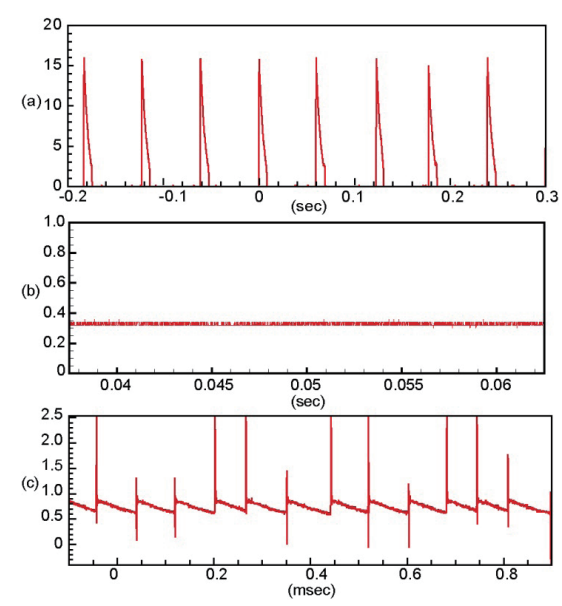

Fig. 4. Voltage drop across the actuator electrodes at various electrode gaps and ballast resistances. The discharge set-point currents are $1 \mathrm{~mA}, 0.32 \mathrm{~mA}$ and $0.7 \mathrm{~mA}$, respectively. current at the set-point value so that it shuts off automatically.

The self-pulsing frequency is almost constant in time at about $16 \mathrm{~Hz}$ and it increases with the discharge setpoint current. Because of the low-frequency pulsing of the discharge current, an audible sound is detected whenever it produces a current peak. Mode (b) exhibits a steady, continuous current flow over time that explains no audible sound wave at a lower set-point current of $0.32 \mathrm{~mA}$ and high ballast resistance of $10 \mathrm{M} \Omega$. In these conditions, the discharge current is predominantly limited by the ballast.

As the discharge current increases, the waveform in Fig. 4 (b) becomes that in (c), where a self-pulsing phenomenon occurs again. However in this regime, the self-pulsing frequency is about 10 to $20 \mathrm{kHz}$, which is three orders of magnitude higher than in mode (a). This explains the highfrequency audible noise from the discharge in mode (c). The discharge current exhibiting a saw-tooth waveform remains above a certain value close to the set-point current of 0.7 $\mathrm{mA}$, while in mode (a), the discharge current remains zero between pulses. Vertical jumps in the discharge current at the rise time of each pulse occur due to electromagnetic signal noise from the electrical connections.

Figure 5 shows the supply voltage (the total voltage drop across the electrodes and ballast resistors) and the discharge voltage (the voltage drop across the electrodes) at different ballast resistances from $1 \mathrm{k} \Omega$ to $20 \mathrm{M} \Omega$. The discharge current is set constant at $0.5 \mathrm{~mA}$ for each case with a 2.5$\mathrm{mm}$ electrode gap distance. It is clear that higher supply voltage is required at higher ballast to provide the same setpoint current. However, the discharge voltage is more or less constant regardless of the ballast resistance. This implies that the discharge regime shown in Figs. 3 and 4 is weakly related to the supply voltage.

A linear momentum increase is achieved by $F=q E$ (where

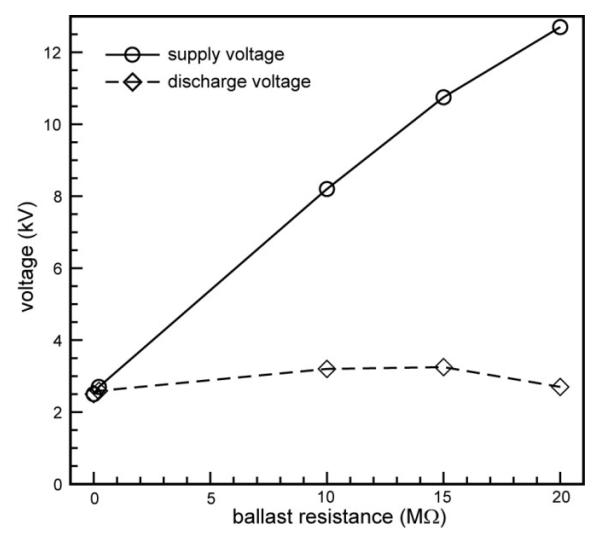

Fig. 5. Supply voltage (solid line) and the discharge voltage between the electrodes (dashed line) at different ballast resistances. The discharge current is set constant at $0.5 \mathrm{~mA}$ for each case. 
$\mathrm{q}$ is the space charge density $\left(\mathrm{C} / \mathrm{m}^{3}\right), E(=-\nabla \Phi)$ is the electric field, and $\Phi$ is the electrical potential). Based on this, it is reasonable to expect the actuation effect by the discharges with various resistances to be similar because of the similar discharge voltages with the same discharge current. As a result, the space charge density is unchanged. In other words, the induced flow speed is expected to increase as the discharge current increases because of more particles colliding with neutrals at higher current density. However, the actuation effect by the plasma actuator was determined by both the discharge current and the discharge modes. As shown in Fig. 2, the actuation effect can occur in an opposite manner to that expected. In this case, the achieved flow actuation may not be caused by the increase in linear momentum. It must be considered that the gas heating effect generally becomes significant at higher currents.

\subsection{Flow actuation characteristic}

Figure 6 shows Schlieren images of the induced flow fields driven by the plasma actuator with different discharge modes. Fig. 6 (a) corresponds to the case of lower ballast resistance presented in Fig. 3 (a), while Figs. 6 (b) and (c) correspond to the higher ballast conditions presented in Figs. 3 (b) and (c), respectively. Interestingly, the induced flow fields driven by the plasma actuators in different discharge modes exhibit very different structures, even though the discharge currents and voltages are not very different from each other. Fig. 6 (a) shows semicircular wave structures similar to those seen in synthetic jets. It starts with an oval shape between the electrodes and grows bigger and becomes circular until it slowly diffuses

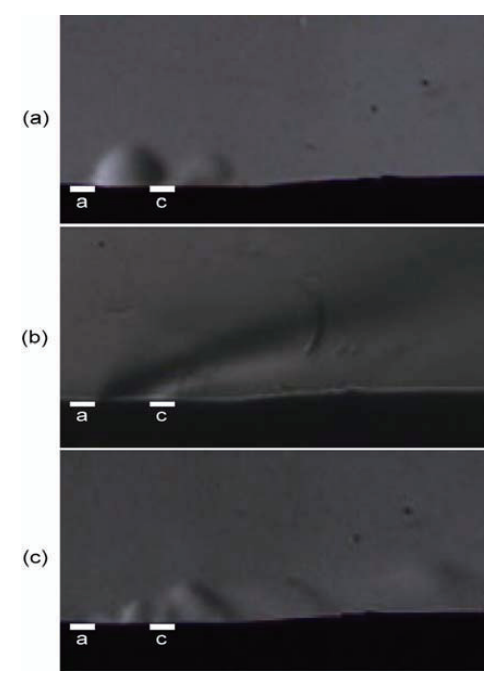

Fig. 6. Schlieren images of induced flow field produced by the plasma actuator in different discharge modes. 'a' denotes an anode and ' $c$ ' denotes a cathode. and vanishes without having any specific propagation direction. In these conditions, the Schlieren image reveals pulsed behavior, as observed in Fig. 4 (a).

Since the set-point current is still low at $1 \mathrm{~mA}$, it is expected that a linear momentum increase will have an effect. However, the flow field in Fig. 6 (a) implies that the actuation is not predominantly driven by the linear momentum. Instead, the gas heating effect seems to play a dominant role in this discharge mode. The pulse frequency increases as the discharge current increases. The gas heating effect near the electrodes also increases with the discharge current so that the effect of induced directional momentum is further degraded due to a higher buoyancy effect.

At higher ballast and lower current of about $0.5 \mathrm{~mA}$ or less, the induced flow field turns very stable and exhibits a straight wave structure, as shown in Fig. 6 (b). This is generally expected to occur in DC discharges with low current (several $\mathrm{mA}$ or less). The Schlieren image is consistent with Fig. 3 (b), where the discharge is stable without audible noise. It is clear that a linear momentum increase is dominant over a gas heating effect in this discharge mode. As the discharge current further increases to near $1 \mathrm{~mA}$ or above, the induced flow field turns into continuously repeating jets with high frequencies and flowing in a tangent direction, as shown in Fig. 6 (c). Again, the gas heating effect does not seem to be dominant in this discharge mode, but its effect starts to increase with an increasing current jump at each pulse at higher currents.

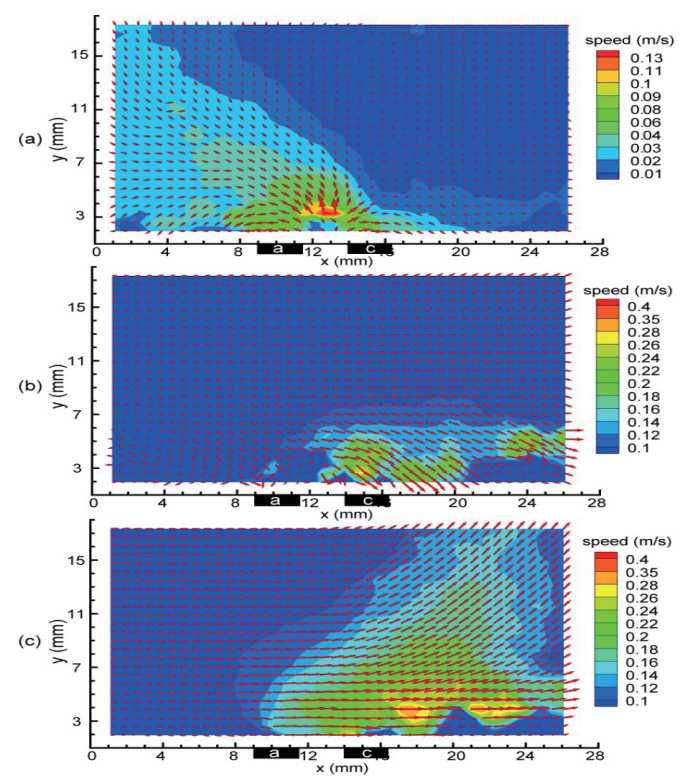

Fig. 7. Vector fields of the induced flow obtained by particle image velocimetry (PIV) technique. (a), (b) and (c) correspond to the mode (a), (b) and (c), respectively, as shown in the previous figures. 'a' denotes an anode and ' $c$ ' denotes a cathode. 
Detailed flow structures are obtained by the PIV technique. Fig. 7 shows the vector fields of the induced flow velocity in different discharge modes. The top image corresponds to mode (a). As expected from the Schlieren image of Fig. 6 (a), the vector fields indicate that an induced flow expands in every direction, even though the discharge current is aligned in the horizontal direction or curved between the anode and cathode. This implies that the gas heating effect is dominant over that of the linear momentum increase. This can be true when the continuous current turns into very short pulsed DC current, even with a low current. This is also the case with a nano-second pulsed dielectric-barrier discharge plasma actuator, where fast gas heating is achieved through dissociation of repulsive

As shown in Fig. 4 (a), the discharge in mode (a) exhibits pulsed operation with current overshoots. The peak current level is still not high enough to cause a significant gas heating effect, but a rapid increase in the current may cause effective gas heating and provide the discharge with high $E / n$. In addition, the pulse-on time is relatively short to impart sufficient linear momentum to stagnant ambient air.

The middle and bottom images correspond to the cases of Figs. 3 (b) and (c), respectively. In both cases, an induced flow is directed toward the cathode with similar velocity magnitudes. In Fig. 7 (b), where the discharge current exhibits a continuous waveform, the induced flow is directed toward the cathode with a slope that implies that the current lines are possibly curved near the cathode region. This is especially possible in a Townsend discharge, where the plasma potential gradually increases near the cathode. In Fig. 7 (c), the induced flow direction is more parallel to the surface than in Fig. 7 (b), which implies the current lines are flatter and the actuation effect is bigger with the same velocity magnitude.

\section{Conclusion}

As shown in Fig. 7, the actuation effect can differ noticeably depending on the discharge modes, even at similar set-point discharge currents and discharge voltages. The discharge produced by the plasma actuator can operate in either continuous or self-pulsed DC, depending on the current levels and ballast resistances, even though a continuous DC power input is provided. The self-pulsed discharge can produce either an isotropic gas expansion effect at lower ballast resistances or a directional linear momentum effect at higher ballast resistances. This implies that a gas heating effect can be dominant over the effect of the linear momentum increase in discharge mode (a).
The self-pulsed discharge with higher ballast resistances produces an induced flow parallel to the actuator surface near the cathode area.

The frequency of audible sound is proportional to the frequency of the discharge pulse. The continuous discharge that can be obtained with lower current and higher ballast resistances can provide stable flow actuation with curved velocity vectors in a direction from the anode to cathode. From the I-V characteristics of mode (b) and mode (c), it can be concluded that it is necessary to provide a continuous DC component in the discharge current to achieve a linear momentum increase against a gas heating effect. In other words, a greater gas heating effect can be achieved with a pulsed discharge without a DC component. This gives an idea that the active flow control can be achieved only by switching discharge regimes under a fixed supply power, and various actuation capabilities can be obtained when the discharge is carefully operated by controlling the frequency and discharge current.

\section{Acknowledgement}

This work was supported by the 2013 Research fund of the University of Ulsan.

\section{References}

[1] Yoo, D. W., Won, D. Y. and Tahk, M. J., "Optical flow based collision avoidance of multi-rotor UAVs in urban environments", International Journal of Aeronautical and Space Science, Vol.12, No. 3, 2011, pp.252-259.

[2] Mueller, T. J., "Aerodynamic measurements at low Reynolds numbers for fixed wing micro-air vehicles", RTO AVT/VKI Special Course on Development and Operation of UAVs for Military and Civil Applications, 1999.

[3] Najafi, Y., Design of a High Altitude Long Endurance Solar Powered UAV, M.S. Thesis, San Jose State University, 2011.

[4] Santhanakrishnan, A., Pern, N. J., Ramakumar, K., Simpson, A. and Jacob, J. D., "Enabling flow control technology for low speed UAVs", AIAA Infotech@Aerospace, Arlington, Virginia, USA, 2005, AIAA 2005-6960.

[5] Selig, M. S., "Low Reynolds Number Airfoil Design", VKI Lecture Series - Low Reynolds Number Aerodynamics on Aircraft Including Applications in Emerging UAV Technology, 2003, RTO/AVT-VKI-104.

[6] Pelletier, A. and Mueller, T. J., "Low Reynolds number aerodynamics of low-aspect-ratio, thin/flat/cambered-plate 
wings", Journal of Aircraft, Vol. 37, No. 5, 2000, pp.825-832.

[7] Sohn, M. H. and Chung, H. S., "Control of delta-wing vortex by apex strake”, KSAS International Journal, Vol. 8, No. 2, 2007, pp.98-106.

[8] Blackwelder, R. F. and Gad-el-Hak M., "Method and apparatus for reducing turbulent skin friction", US Patent US 4932612 A, 1990.

[9] Godard, G. and Stanislas, M., "Control of a decelerating boundary layer Part 1: Optimization of passive vortex generators", Aerospace Science and Technology, Vol. 10, 2006, pp.181-191.

[10] Ruffin, S. M., Gupta, A. and Marshall, D., "Supersonic Channel Airfoils for Reduced Drag”, AIAA Journal, Vol. 38, No. 3, 2000, pp.480-486.

[11] Saric, W. S. and Reed, H. L., "Supersonic Laminar Flow Control on Swept Wings Using Distributed Roughness", 40th AIAA Aerospace Sciences Meeting and Exhibit, Reno, NV, USA, 2002, AIAA 2002-0147.

[12] Oruc, V., "Passive control of flow structures around a circular cylinder by using screen", Journal of Fluids and Structures, Vol. 33, 2012, pp.229-242.

[13] Kral, L. D., "Active flow control technology", ASME Fluids Engineering Division Technical Brief, 2000.

[14] Jahanmiri M.. "Active flow control: A review", Research report, 2010, ISSN 1652-8549.

[15] Lee, K. Y., Chung, H. S. and Cho, D. H., "A PIV Study of Flow Patterns Over Stationary and Pitch-Oscillating Airfoils with Blowing Jet", KSAS International Journal, Vol. 9, No. 1, 2008, pp. 111-120.

[16] Sinha, S. K. and Ravande, S. V., "Drag Reduction of Natural Laminar Flow Airfoil with a Flexible Surface Deturbulator", 3rd AIAA Flow Control Conference, San Francisco, CA, USA, 2006, AIAA 2006-3030.

[17] Cho, Y. C. and Shyy, W., "Adaptive flow control of low-Reynolds number aerodynamics using dielectric barrier discharge actuator", Progress in Aerospace Sciences, Vol. 47, 2011, pp.495-521.

[18] Moreau, E., "Airflow control by non-thermal plasma actuators", Journal of Physics D: Applied Physics, Vol. 40, No. 3, 2007, pp. 605-636.

[19] Benard, N., Jolibois. J., Mizuno, A. and Moreau, E., "Innovative Three-Electrode Design for Definition of Multiple Dielectric Barrier Discharge Actuators", ESA/IEEEIAS/IEJ/SFE Joint Conference on Electrostatic, Boson, USA, 2009.

[20] Huang, J., Corke, T. C. and Thomas, F. O., "Plasma Actuators for Separation Control of Low-Pressure Turbine Blades", AIAA Journal, Vol. 44, No. 1, 2006, pp. 51-57.

[21] Jukes, T., Segawa, T. and Walker, S., "Active Separation Control over a NACA0024 by DBD Plasma Actuator and FBG
Sensor", Journal of Fluid Science and Technology, Vol. 7, No. 1, 2012, pp. 39-52.

[22] Vorobiev, A., Rennie, R. M. and Jumper, E. J., "Lift Enhancement by Plasma Actuators at Low Reynolds Numbers", 5th AIAA Flow Control Conference, AIAA 2010483.

[23] Rethmel, C., Little, J., Takashima, K. Sinha, A., Adamovich, I. and Samimy, M., "Flow Separation Control over an Airfoil with Nanosecond Pulse Driven DBD Plasma Actuators", AIAA Journal, AIAA 2011-487.

[24] Menghu, Han, Jun, L., Zhongguo, N., Hua, L., Huangyin, Z. and Weizhuo, H., "Aerodynamic performance enhancement of a flying wing using nanosecond pulsed DBD plasma actuator", Chinese Journal of Aeronautics, Vol. 28, 2015.

[25] Tabatabaeian, S., Mirzaei, M., Sadighzadeh, A., Damideh, V. and Shadaram, A., "Experimental investigation of the effects of various plasma actuator configuration on lift and drag coefficients of a circular cylinder including the effects of electrodes", Chinese Journal of Aeronautics, Vol. 25, 2012, pp.311-324.

[26] Klimov, A., Moralev, I., Bityurin, V.A., Kazansky, P.N., Chertov, D.A. and Borisov I.A, "Flow Around Wing Model with a Surface HF Discharge", 49th AIAA Aerospace Sciences Meeting including the New Horizons Forum and Aerospace Exposition, Orlando, FL, USA, 2001, AIAA 2011-1146.

[27] Porter, C. O., Baughn, J. W., McLaughlin, T. E., Enloe, C. L. and Font, G. I., "Temporal Force Measurements on an Aerodynamic Plasma Actuator", 44th AIAA Aerospace Sciences Meeting and Exhibit, AIAA 2006-104.

[28] Moreau, E., Sosa, R., and Artana, G., "Electric wind produced by surface plasma actuators: a new dielectric barrier discharge based on a three-electrode geometry", Journal of Physics D: Applied Physics, Vol. 41, No. 11, 2008, 115204 (12 pp).

[29] Ye, Q., Wu, Y., Li, X., Chen, T. and Shao, G., "Uniformity of dielectric barrier discharges using mesh electrodes", Plasma Sources Science and Technology, Vol. 21, No. 6, 2012, 065008 (7 pp).

[30] Grange, F., Soulem, N., Loiseau. J. F. and Spyrou, N., "Numerical and experimental determination of ionizing front velocity in a DC point-to-plane corona discharge," Journal of Physics D: Applied Physics, Vol. 28, No. 8, 1995, pp. 1619-1629.

[31] Shin, J. and Raja, L. L., "Cathode-sheath driven lowspeed aerodynamic flow actuation using direct-current surface glow discharges", Journal of Electrostatics, Vol. 68, No. 5, 2010, pp. 453-457.

[32] Sosa, R. and Artana, G., "Steady control of laminar separation over airfoils with plasma sheet actuators", Journal 
of Electrostatics, Vol. 68, No. 7-9, 2006, pp. 604-610.

[33] Roth, J. R., "Aerodynamic flow acceleration using paraelectric and peristaltic electrohydrodynamic effects of a one atmosphere uniform glow discharge plasma", Physics of Plasmas, Vol. 10, No. 5, 2003, pp. 2116-2126.

[34] Stark, R. H. and Schoenbach, K. H., "Direct current high-pressure glow discharges", Journal of Applied Physics,
Vol. 85, No. 4, 1999, pp. 2075-2080.

[35] Raizer, Yu. P., Gas Discharge Physics, Springer, New York, 1991.

[36] Roupassov, D. V., Nikipelov, A. A., Nudnova, M. M. and Starikovskii, A. Yu., "Flow separation control by plasma actuator with nanosecond pulsed-periodic discharge," AIAA Journal, Vol. 47, No. 1, 2009, pp. 168-185. 